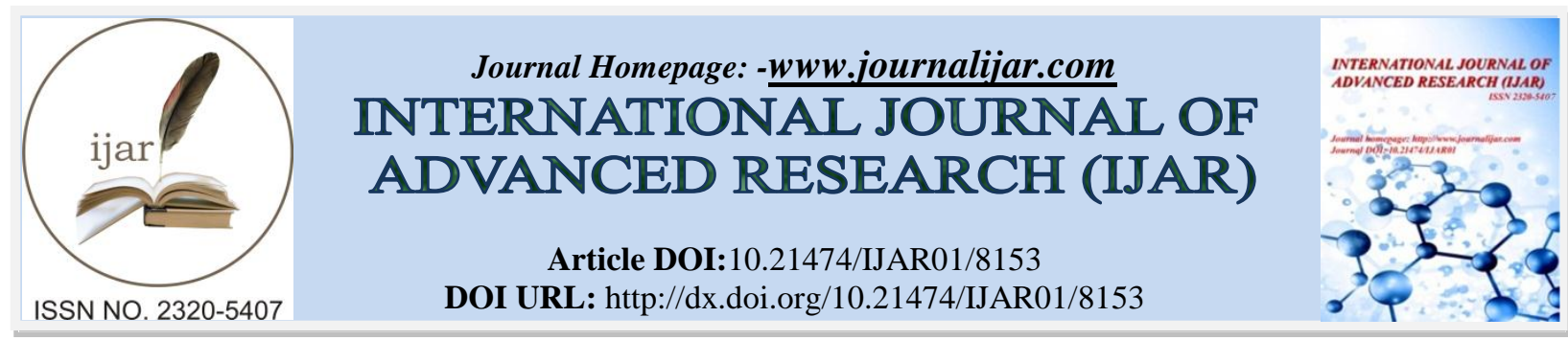

RESEARCH ARTICLE

\title{
COMPARITIVE STUDIES OF VARIENT DIETS IN DROSOPHILA MELANOGASTER WITH RESPONSE TO GUSTATION AS FEEDING BEHAVIOUR.
}

\author{
B.K Neethu, Choudhury.A and Chanda.P. \\ School of Sciences, Department of Life science,Garden city University,Bengaluru560049.
}

\section{Manuscript Info}

Manuscript History

Received: 04 October 2018

Final Accepted: 06 November 2018

Published: December 2018

Keywords:

Drosophila melanogaster,Gustatory,

Diets.

\begin{abstract}
Gustatory assay and nutritional constituents is an important factor determning the feeding behaviour of an organism. In this review, feeeding behaviour in Drosophila melanogaster pre-adult (larvae) was investigated and the results were compared with those for post- adult (flies) stages, supplementing Drosophila with varient diets namely yeasts and fruits. It was obserevd that pre-adult preferred yeast supplemented diets rather than control and fruits, while the post adult showed preference choice for fruits than yeast and control. Thus, higher nutritional value of fruit and yeast with control is expected to favor sensory and physiological adaptations for feeding behaviour, showing gustatory receptors act cooperatively in larval and adult taste sensing in every stages of development having different degree of responsiveness to varient diet supplemented.
\end{abstract}

Copy Right, IJAR, 2018,. All rights reserved.

\section{Introduction:-}

Behaviours such as eating, mating, and sleeping that are observed in humans are also seen in Drosophila. Therefore, the possible effect of genetics upon human behaviour can also be assessed. Insects such as the fruit fly, Drosophila melanogaster, rely on contact chemo sensation to detect nutrient-rich foods, to avoid consuming toxic chemicals, and to select mates and hospitable zones to deposit eggs. Flies sense tastants and non-volatile pheromones through gustatory bristles and pegs distributed on multiple body parts including the proboscis, wing margins, legs, and ovipositor. In Drosophila, the taste neurons located in sensilla in several body regions sense and distinguish nutritive substances such as sugars, amino acids, and low salt, and potentially harmful ones such as high salt, acids, sugar and a diverse variety of bitter compounds (Freeman and Dahanukar, 2015).

Drosophila melanogaster has emerged as a tractable model organism for studying a number of biological phenomena including feeding behavior's under various conditions (Sokolowski, 2001). At times, Drosophila larvae may encounter nutritional stress that could be transient or chronic and therefore must learn to adapt to it for survival. Remarkably, this species has evolved to adapt to ephemeral as well as persistent nutritional stress (Vijendravarma et al., 2012a). An earlier report suggested that larval malnutrition significantly affects foraging behavior in Drosophila (Vijendravarma et al., 2012b).

The decision by insects to initiate courtship and mating, lay eggs or consume plants and other foods is influenced heavily by direct contact with non-volatile compounds. Mammals and insects such as the fruit fly, Drosophila melanogaster, respond to many of the same tastants, including sugars and bitter compounds. However, in contrast to mammals, which taste primarily through taste buds on the tongue, fruit flies are able to sample tastants through

Corresponding Author:-B.K Neethu.

Address:-School of Sciences, Department of Life science,Garden city University,Bengaluru560049 
receptor cells on many parts of the body. The wide distribution of taste organs contributes to the various behaviors initiated by gustatory input and to animal survival (Sanchez et al.,2009).

Drosophila and many other insects detect tastants on multiple body parts through hair like projections referred to as gustatory sensilla. The proboscis includes both external and internal sensilla and might be considered the functional equivalent of the mammalian tongue. Thirtyone external sensilla are situated in four rows on each of two labelialpalps or labella, along with shorter and simpler taste structures, the taste pegs. The internal sensilla in the pharyngeal portion of the proboscis permit the flies to evaluate food immediately following ingestion but before it is transferred to the digestive system. Sensilla on the labella, anterior wing margins and legs enable the flies to sample potential foods without consuming them. This ability contributes to survival since the animals can taste attractive nutrients or toxic chemicals before deciding whether or not to extend the proboscis and ingest the food (Singh,1997).

Yeasts and other micro-organisms occupy a commonly overlooked trophic level between plants and insect herbivores. Insects from several orders, including beetles, flies, ants and bees interact with yeasts (Jansonet al.,2008). Many insects feeding on flowers or fruit encounter yeasts (Ganter 2006) and yeasts that use insects as hosts and vectors are widespread (Suhet al.,2005; Gibson and Hunter 2010). For Drosophila flies, the presence of yeast in larval diet substrates is fundamental for the occupation of niches and separation of species, and interactions between yeast and fruit flies have led to mutual co-adaptations. Nutritional gains or detoxification of harmful chemicals sustain larval development; while feeding and vectoring by flies mediate dispersal and outbreeding of yeast strains (Bell and Greig2007).

Insects use chemical cues to identify host plants, which suggests that chemosensory perception could be a target of natural selection during host specialization, because adult female Drosophila must identify and lay eggs on prospective host plants for their offspring that subsequently feed on the microbes living on rotting plant tissue, the discovery of those factors that influence host selection is essential to understanding host specialization. However, basic knowledge regarding D. melanogaster's chemical ecology and olfactory preferences are currently lacking (Rueben Bauer, Schlyter, Hansson, Lofstedt, \& Larsson, 2008). Against this backdrop, we conducted a simple experiment with the polyphag D. melanogaster, a known food / host generalist but close relative of several specialist species (Whiteman \& Pierce, 2008), to look for evidence of mild fruit (host) preferences upon which natural selection could work by releasing large numbers of flies into a chambered apparatus with a different fruit sample in each chamber (apples, bananas, oranges and an empty control).

The model organisms Drosophila melanogaster and S. cerevisiae are particularly suitable experimental models for studying the co-evolution of yeasts and insects. D. melanogaster is attracted to overripe fermenting fruit for feeding, mating and oviposition (Ganter, 2006). Yeasts are known to support larval development and survival (Anagnostou et al.,2010).

A fundamental issue towards an understanding of the mechanisms driving insect-fruit-yeast relationships is the individual contribution of the plant substrate and the yeast to habitat quality and habitat choice by the insect. It has so far not been investigated whether attraction and oviposition of D. melanogaster are triggered by the fruit, the yeast or the combination of both. In addition, it is unclear whether volatiles released by fruit and yeast correlate with the nutritional value and suitability of the substrate for larval development.

The gustatory system allows animals to detect chemical compounds in the environment and determine their value as potential food sources. To make this assessment, animals detect two different features of taste stimuli with the gustatory system: the concentration and the quality of a taste compound. In humans, taste concentration is perceived as intensity and taste quality as a component of flavor. Determining how these two features of taste stimuli are encoded by the nervous system and used to direct behavior is central for the neural basis of taste perception. The above study is performedtocomparethe feeding behaviour of variant diets in Drosophila melanogasterpre-adult (larvae) and post- adult (flies)in response to taste choice on supplementation to variant diets by gustatory assay as feeding behaviour.

\section{Materials and method:-}

Drosophila melanogaster stock was obtained from Drosophila stock were routinely cultured in standard wheat cream agar medium. From this stock about 200-250 eggs were collected and placed in culture bottles (about 10 to 50 eggs/bottle). The newly hatched flies from these stocks were considered to be the parental stock (Harini , 2011). 
About 30 males and females were separated by gender and were transferred to the fresh media containing variable concentrations of diets namely dry yeast, granular yeast $(0.5 \mathrm{mg} / 1 \mathrm{mg}, 1.5 \mathrm{mg} / 100 \mathrm{ml}$ respectively) and orange , pineapple $(10 \mathrm{ml}, 20 \mathrm{ml}, 30 \mathrm{ml} / 100 \mathrm{ml}$ respectively) along with the control.

\section{Gustatory assay}

The method described by Lee et al. 2010.was adopted for gustatory assay. Larva and adult flies were reared for 20 or 40 days in media supplemented with $10 \%$ PJ or $200 \mu \mathrm{M} \mathrm{RV}$. A basal media control was also maintained. To perform a feeding assay, after starving the flies for $2 \mathrm{~h}, 30$ male or female flies from each experimental group were transferred into the vials containing the specific diets with bromophenol blue dye $(0.05 \% \mathrm{wt} / \mathrm{vol})$.After $10 \mathrm{~min}$ of feeding, the fed flies were etherized, washed with phosphate-buffered saline (PBS), and homogenized in $1 \mathrm{ml}$ of distilled water. The absorbance of 100 times diluted supernatant was measured at $595 \mathrm{~nm}$ using a spectrophotometer

\section{Larval feeding assay:}

Larva was feeded for 15 minutes. After feeding the alimentary canal of larva got bluish in color. Feeded larva was taken and washed with PBS buffer. After washing homogenized it with glass rod in appendrops which contained 1 $\mathrm{ml}$ of distil water. Following the homogenization, after-that centrifugation was done for 10 minutes at $3000 \mathrm{rpm}$. Collected the supernatant and diluted all the tubes with $10 \mathrm{ml}$ of distil water. values were measured by colorimeter at $595 \mathrm{~nm}$.

\section{Adult feeding assay:}

Followed with adult flies (male and female) with same concentration. Flies were anesthetized to be sorted, sexed and scored. We used 26 bottles (12 bottles for male and 12 bottles for females and 2 for control) instead of petriplates and after pouring media we leaved 1 hour for cooling but rest of the assays were performed similarly as described above for the larval feeding.

\section{Statistical analysis}

The total larvae and flies in each of the experimental group survived was calculated by standard deviation with the mean value and standard error.

\section{Result:-}

To determine the feeding behaviour in Drosophila melanogaster, the media was supplemented with $0.1,1$ and $5 \mathrm{mg}$ of dry yeast and 10, 20 and $15 \%$ of fruit juice along with control where, $n=30$. The mean and standard error was calculated for yeast (dry yeast and granular yeast)i.e0.5\%, 1\%,1.5\% supplemented diet showed increased mean values with that of the control in larvae Fig. 1 .Between concentration showed higher percentage with increased feeding rate in high concentration compared to low and mid concentration in yeast supplement diet. whilein Fig. 2 and Fig 3,depicts adults (Flies) male and female showed increased feeding behaviour in high concentration and decreased feeding in low concentration along with control.

In fruits supplemented diets (orange and pineapple) i.e.10\%, 20\%, 30\%, larva showed decreased mean value with increased concentrationand adults showed increased mean value with increased concentration as shown inFig.1.When adult male were supplemented with variant diets along with control percentage of gustatory feeding increased in both yeast and fruit fed diets. percentage of gustatory feeding showed increased preference for fruits than yeast with respect to control. High percentage of gustatory feeding was observed in mid concentrations of each supplemented diet with respect to low and high concentration. While the female showed increased mean value in mid concentration than low and high concentrationwith that of control Fig. 2 and Fig 3.

Study was focussed on both the larval and adult (female and male) stages. It was observed that percentage of larvae fed on yeast concentrations showed increased feeding behaviour than the fruits supplemented to them compared to that of control. In adult both male and female when fed with yeast and fruits it was observed that they showed the increased feeding behaviour for fruits supplemented than yeast compared to the control. Showing innate preferences to these compounds differ respectively.Amount of yeast diet fed by larvae increased with concentration compare to the adults and adults fed more on fruit diet compare to larva. 


\section{Discussion:-}

The limited discrimination of the fly taste system is quite different from the discrimination seen in the fly olfactory system. Several olfactory associative learning paradigms have demonstrated exquisite discrimination of different odors (Berry et al.,2008). Flies associate an odor paired with a reward or a punishment, and, in general, this association is specific to the paired odor. Both the identity of the individual compound and the concentration may be used to distinguish an odor, with concentration discrimination occurring in situations in which quality information is not accessible to the fly The Drosophila gustatory system provides an attractive model for studies of taste discrimination because it is an experimentally tractable system that retains similarities to mammalian taste. Like mammals, Drosophila distinguishes different taste concentrations, with dose-dependent preference and avoidance behaviours. These flies also distinguish a few taste qualities, including sugar and bitter tastes, and possess modalityspecific, peripheral taste cells (i.e., sugar-selective and bitter-selective neurons) (Xia and Tully, 2007).

When larva were supplemented with variant diets along with control percentage of gustatory feeding increased in both yeast and fruit fed diets, percentage of gustatory feeding showed increased preference for yeast than fruits with respect to control. High percentage of gustatory feeding was observed in mid concentrations of each supplemented diet with respect to low and high concentration.

The time taken to develop from egg to adult lasted for 6 days in the flies raised on the high protein diet, while flies which were raised on the low protein diet had a significantly longer metamorphic stage (9-11days). Hence one can conclude that the enrichment of protein concentration would yield faster metamorphosis (Neethu et el.,2014) Taste behaviors are robust and innate, with sugars mediating acceptance and bitters mediating avoidance. Here, we show that taste behaviors may be modified by conditioning, because sugars (or sugars plus bitter compounds) become less acceptable when paired with a noxious stimulus. This conditioning assay provided the opportunity to examine the tastes that a fly discriminates.

The ability to associate a taste compound with a unique behavior allowed us to as test whether learned associations are based on intensity or quality. In the simplest scenario, when two concentrations of the same sugar were tested, flies learned to avoid the concentration paired with heat. This association generalized to lower concentrations (less palatable) but not to higher concentrations (more palatable). Experiments testing different sugar pairs or bitter pairs showed similar results, with flies generalizing associations to less palatable compounds but not to more palatable compounds. These results argue that flies discriminate compounds based on intensity but not on chemical identity. Although we cannot rule out discrimination based on chemical structure with other taste compounds or a different behavioral assay, our data support the notion that the salient feature flies discriminate is palatability or "sweeter" (Berry, 2008).

Feeding behavior requires conciliation, whereby individuals have to ransom some nutritional needs to satisfy those that have high regulatory priority. As a result, there is a prerequisite for an individual to consume protein, carbohydrates, or amino acids to proclaim its total calories to meet its essentials.

Feeding at the pre-embryonic level certainly has an impact on further development of the post-embryonic stages. In consistent the post-embryonic stages influence many adult traits, such as body size and fitness determinants. In response to this, the present study is well apprehended with the dietary enrichment enforcing the rate of development, life history traits and adult body size (Neethu and Harini, 2017). According to the result observed our study reveals that Drosophila melanogaster adults attracts more sweeter diets than compared to yeasts under in vitro conditions.

\section{Conclusion:-}

The hypothesized results were that the larva would prefered the yeast extract over the other food choices and adults preferred fruit extract over the yeast extract. With respect to the standard deviation, more trials were conducted to form a stronger conclusion. The remarkable increase in number and sophistication of studies on various Drosophila sensory systems over the last few years has led to a more comprehensive and detailed understanding of how the fruit fly perceives its world and processes sensory stimuli. Effect on specific taste modalities has shown the preferences of the Drosophila melanogaster both larval and adult stages where, each developing stages shows different preference with the choice of taste. 


\section{Acknowledgments:-}

The authors thanks Dr.Joseph V.G, the Honary consultant of the Republic Maldives in Bangalore and Chancellor Garden City University for his encouragementand laboratory support to carry out the above work. We would also like to thank Vice-chancellor, Principal, HOD and all faculty members of School of Sciences, Department of Life Sciences-Garden City University, Bangalore for all their support.

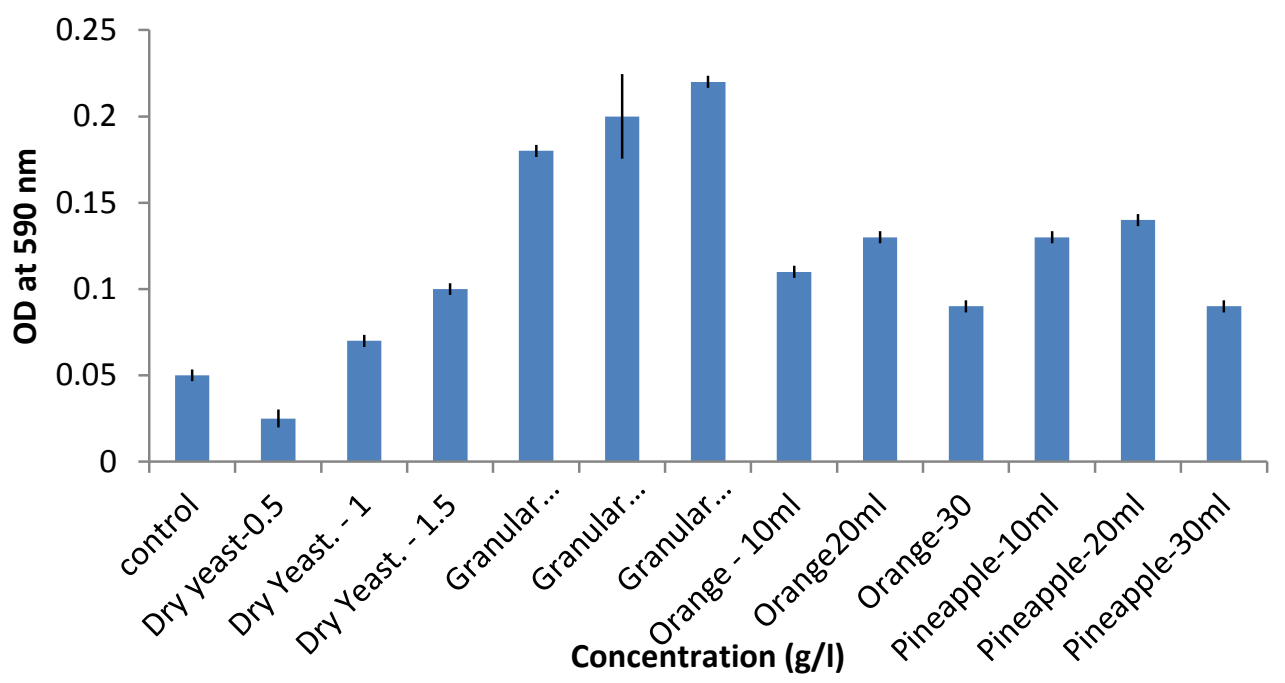

Fig 1:-Mean ( \pm ) gustatory feeding assay in larva of Drosophila melanogasterwhen supplemented with varied diets.

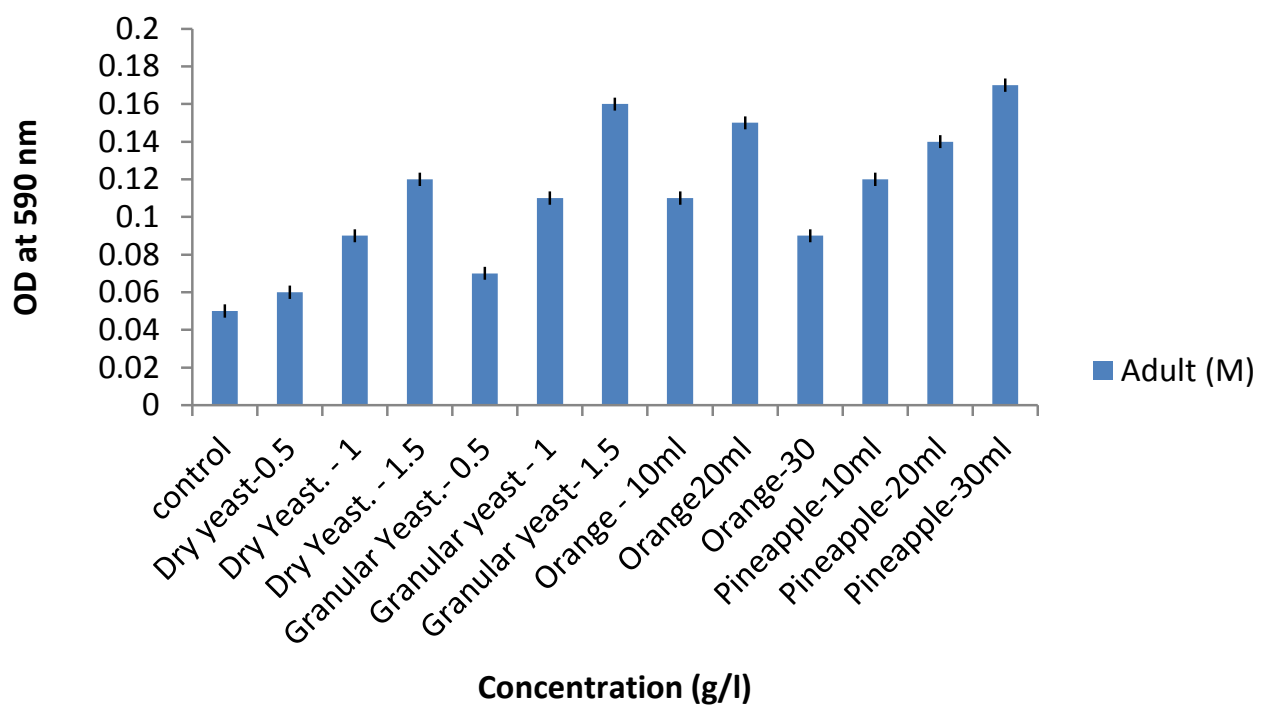

Fig 2:-Mean ( \pm ) Gustatory feeding assay in adults (male)Drosophila melanogaster when supplemented with variant diet. 


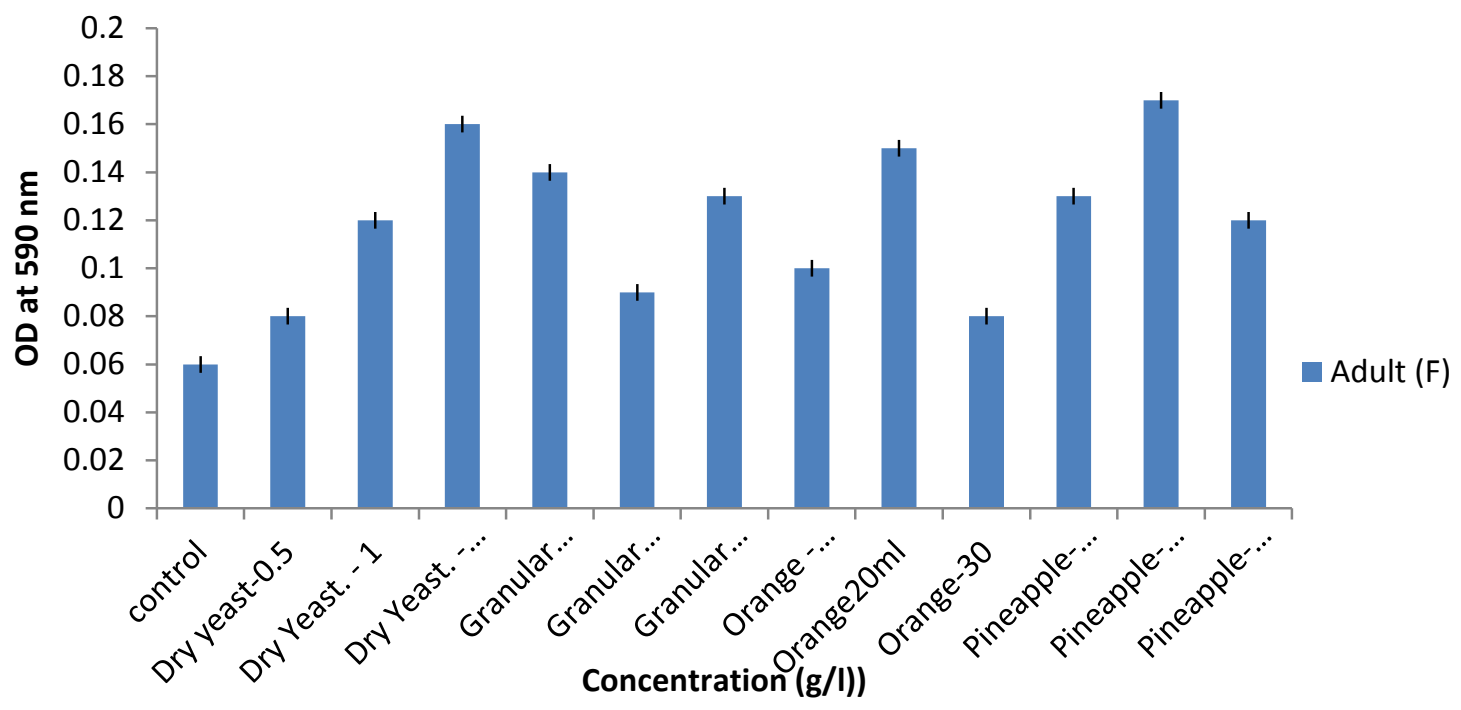

Fig 3:-Mean ( \pm ) Gustatory feeding assay in adults female Drosophila melanogasterwhen supplemented with variant diet.

\section{References:-}

1. Freeman, E.G.andDahanukar, A. (2015). Molecular neurobiology of Drosophila taste. Current Opinion in Neurobiology. 34,140-148.

2. Sokolowski, M.B., (2001). Drosophila: genetics meets behaviour. Nature reviews. Genetics, 2(11), p.879.

3. Vijendravarma, R.K., Narasimha, S. and Kawecki, T.J., (2012a). Chronic malnutrition favours smaller critical size for metamorphosis initiation in Drosophila melanogaster. Journal of evolutionary biology, 25(2), pp.288292.

4. Vijendravarma, R.K., Narasimha, S. and Kawecki, T.J., (2012b). Evolution of foraging behaviour in response to chronic malnutrition in Drosophila melanogaster. Proceedings of the Royal Society of London B: Biological Sciences, 279(1742), pp.3540-3546.

5. Janson,E.M.,Stireman,J.O.III,Singer,M.S.andAbbot,P.(2008).Phytophagous insect-microbe mutualisms and adaptive evolutionary diversification.Evolution.62,997-1012.

6. Suh, S.O., McHugh, J.V., Pollock, D.D. \& Blackwell, M. (2005). The beetle gut: a hyperdiverse source of novel yeasts. Mycological Research, 109, 261-265.

7. Gibson, C.M. and Hunter, M.S. (2010) Extraordinarily widespread and fantastically complex: comparative biology of endosymbiotic bacterial and fungal mutualists of insects. Ecology Letters, 13, 223-234.

8. Reuter, M., Bell, G. \&Greig, D. (2007) Increased outbreeding in yeast in response to dispersal by an insect vector. Current Biology, 17, R81-R83.

9. Whiteman, N. K., \& Pierce, N. E. (2008). Delicious poison: genetics of Drosophila host plant preference. Trends in Ecology \& Evolution, 23(9), 473-478.

10. Ruebenbauer, A., Schlyter, F., Hansson, B., Lofstedt, C., \& Larsson, M. (2008). Genetic Variability and Robustness of Host Odor Preference in Drosophila melanogaster. Current Biology, 18(18), 1438-1443.

11. Anagnostou,C.,Monika,D., and Marko.R. (2010).Influence of dietary yeasts on Drosophila melanogaster life-history traits.EntomologiaExperimentalisetApplicata 136, 1-11.

12. Harini, B.P. (2011). Varaition in life history traits in few members of immigransspecies group of Drosophila exposed to light and dark cycle. Bioscan, 6(1), pp.157-162.

13. Lee, Y., Kim, S.H. and Montell, C.(2010). Avoiding DEET through insect gustatory receptors. Neuron, 67(4), pp.555-561.

14. Berry, J., Krause, W.C., Davis, R.L.(2008). Olfactory memory traces in Drosophila. Prog Brain Res. 169,293304.

15. Xia, S,and Tully, T.(2007) Segregation of odor identity and intensity during odor discrimination in Drosophilamushroom body. PLoSBiol 5:e264. 
16. Neethu,B.K., and Harini,B.P.(2017).Genetic analysis of dietary restrictions in immigrans species group of Drosophila.Thesis.Bangalore University.

17. B.K. Neethu, Y. Ramesh Babu and B.P. Harini (2014); Enhanced protein diet accelerates the rate of development -A study in Drosophila nasutanasuta Int. J. of Adv. Res. 2 (2). 0.

18. Singh, R.N.(1997). Neurobiology of the gustatory systems of Drosophila and some terrestrial insects. Microsc. Res. Tech.39,547-563.

19. Sanchez-Gracia, A., Vieira, F.G., Rozas, J.(2009). Molecular evolution of the major chemosensory gene families in insects. Heredity.

20. Berry, J., Krause, W.C., Davis, R.L.2008. Olfactory memory traces in Drosophila. Prog Brain Res. 169,293304. 